\title{
Treatment of Pregnancy Induced Hypertension and its Effect on Oxidative Stress
}

\author{
Manjusha Mahakarkar', Swanand Pathak ${ }^{2}$, Savarna Ghugare ${ }^{3}$ and Usha Waghmare ${ }^{4}$ \\ ${ }^{1}$ Department of Obstetrics and Gynaecology, Smt.RadhikabaiMeghe Memorial College of Nursing, Datta \\ Meghe Institute of Medical Sciences (Deemed to be University), Sawangi (Meghe) Wardha, India \\ ${ }^{2}$ Department of Pharmacology, Jawaharlal Nehru Medical College, DattaMeghe Institute of Medical \\ Sciences (Deemed to be University), Sawangi (M), Wardha India \\ ${ }^{3}$ Clinical instructor, Child health nursing department, Smt. RadhikabaiMeghe Memorial College of Nursing, \\ Datta Meghe Institute of Medical Sciences (Deemed to be University), Sawangi (Meghe) Wardha, India \\ ${ }^{4}$ Datta Meghe College of Nursing, Nagpur. India
}

\section{ABSTRACT}

Hypertension is the most common medical problem encountered during pregnancy, complicating 5-10\% of Pregnancies. It is associated with oxidative stress, confirmed by measurement of biomarkers and relevant antioxidant enzymes in the placenta and maternal circulation. In a healthy body, Reactive 0xygen Species and antioxidants remain in balance. When the balance is disrupted toward an overabundance of Reactive 0xygen Species, oxidative stress occurs. Reactive Oxygen Species affect multiple physiological processes from oocyte maturation to fertilization, embryo development and pregnancy. Pre- eclampsia is a leading cause of maternal and paternal morbidity. It is pregnancy specific disease characterized by denovo development of concurrent hypertension and proteinuria. Ojectives: 1. To assess the level of physiological parameters in blood of antenatal mothers with Pregnancy induced hypertension. 2. To monitor the treatment of antenatal mothers of Pregnancy induced hypertension. 3. To correlate the treatment of Pregnancy induced hypertension and its effects on oxidative stress with MDA and SOD estimation in antenatal mothers. Methodology: Pregnant women from AVBRH hospital, Wardha were selected for the study. The study was carried out in the department of OBGY which included total 50 patients of Pregnancy Induced Hypertension (PIH) age group of 20-40 years. Selection cases of PIH were done after assessing for BP > 140/90 mm Hg, proteinurea, edema and within 28-42 weeks of gestation.Result:MDA in pregnancy induced hypertension was $109.09 \pm 24$, in preeclampsia was $130.65 \pm 43.02$ and eclampsia was $141.04 \pm 50.95$. SOD in pregnancy induced hypertension was $3.24 \pm 1$, in preeclampsia was $2.69 \pm 1.34$ and eclampsia was $2.59 \pm 1.33$.Discussion:. Thepresent study was planned to detect lipid peroxidationproducts i.e. MDA and SOD inPIH, pre eclampsia and eclampsia. The lipid peroxidation productlike malondialdehyde (MDA) levels has been measured inplasma of hypertensive pregnant women. It was found thathigher 02 free radical production, evidenced by increase levels of MDA in hypertensive pregnant women. Thepresent study shows that, there is significant differencebetween PIH, pre eclampsia and eclampsia regarding serum MDA.Rise in MDA could be due to increased generation ofROS due to the excessiveoxidative damage generated in the hypertensive patients. These 02 species in turn can oxidize many other importantbiomolecules including membrane lipids. The lipidperoxides \& free radicals may be important in pathogenesisof PIH. In similar previous study was done on pregnant women with pregnancy induced hypertension, it was found at there was a significant increase in erythrocytes MDA levels, activates of SOD, \&t GP level. Conclusion: The present study has shown a significant increase in serum MDA levels, the indicator of lipid peroxidation, in the 3 groups suggest that lipid peroxidation plays a role in the pathogenesis of hypertensive disorders of pregnancy. Increased lipid peroxidation causes increased consumption of antioxidant free radical scavenging system. In PIH, preeclampsia and eclampsia there is an imbalance between lipid peroxides and the antioxidant system.

KEY WORDS: PREGNANCY INDUCED HYPERTENSION, SOD, MDA

\section{ARTICLE INFORMATION}

Received 15th Oct 2020 Accepted after revision 30th Dec 2020 Print ISSN: 0974-6455 Online ISSN: 2321-4007 CODEN: BBRCBA

Thomson Reuters ISI Web of Science Clarivate Analytics USA and Crossref Indexed Journal

\section{Clarivate Analytics}

\section{crossef}

NAAS Journal Score 2020 (4.31)

A Society of Science and Nature Publication,

Bhopal India 2020. All rights reserved.

Online Contents Available at: http//www.bbrc.in/

Doi: http://dx.doi.org/10.21786/bbrc/13.15/55

\section{INTRODUCTION}

Pregnancy is a stressful condition in which many physiological and metabolic functions are altered to a considerable extent. Hypertension is the most common medical problem encountered during pregnancy, complicating $5-10 \%$ of pregnancies. It is associated with oxidative stress, confirmed by measurement of biomarkers and relevant antioxidant enzymes in the

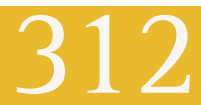


placenta and maternal circulation. In a healthy body, ROS (Reactive Oxygen Species) and antioxidants remain in balance. When the balance is disrupted toward an overabundance of ROS, oxidative stress occurs. ROS affect multiple physiological processes from oocyte maturation to fertilization, embryo development and pregnancy. It also modulates age -related decline in fertility, normal parturition and initiation of preterm labor. Pre- eclampsia is a leading cause of maternal and paternal morbidity. It is pregnancy specific disorder characterised by denovo development of concurrent hypertension and proteinuria.

Need of the Study: Pregnancy is one of the wonderful and noble services imposed by nature. Most of the women may not have many problems during pregnancy, but some are not so lucky, face various problems related to pregnancy and child birth. Every minute, each day somewhere in the world, a woman dies as result of complications related to pregnancy and childbirth. Gestational hypertension and preeclampsia are common disorders during pregnancy with the majority of cases developing at or near term.

Sharma JB et al, 2013A comparative studies was carried out to compare the levels of 3 oxidative stress markers (glutathione peroxidase [GPX], superoxide dismutase [SOD], and malondialdehyde [MDA]) and 2 antioxidants (vitamin $\mathrm{C}$ and lycopene) in healthy and pre-eclamptic pregnant women. Circulating levels of GPX, SOD, MDA, vitamin $\mathrm{C}$ and lycopene were measured in 50 healthy pregnant women and 50 women with pre-eclampsia (PE) (41 with mild PE and 9 with severe PE) attending the antenatal clinic or admitted to the maternity ward of the All-India Institute of Medical Sciences, New Delhi, India.

They were found that the levels of GPX, SOD and MDA were significantly higher in women with PE than in controls, and the increase was higher in women with severe $\mathrm{PE}(\mathrm{P}<0.001 \mathrm{using}$ analysis of variance and the Kruskal Wallis test). The levels of vitamin C and lycopene were significantly lower in women with PE than in controls, with a greater decrease in women with severe PE. Then concluded thatIncreased levels of oxidative stress markers and decreased levels of antioxidants in pre-eclamptic women suggest that oxidative stress markers play a significant role in the pathophysiology of pre-eclampsia, and that supplemental dietary antioxidants may have a beneficial role in the prevention of pre-eclampsia in women at high-risk for this condition.

In this study 154 prescriptions were, a higher number, $55.84 \%$ belonged to stage II hypertension (B.P . $\geq 160 / \geq$ 100 ) before administration of drugs. Out of 154 patients, 123 (i.e, 79.87\%) received a single antihypertensive drug while 27 (i.e, $17.53 \%$ ) received two antihypertensive drugs and 4 patients (2.59\%) received three drugs. Methyl dopa was the most common antihypertensive drug used as monotherapy in 54 (i.e, 35.06\% cases), followed by Labetalol in 32 (i.e, 20.77\%) cases. Combination of Methyl dopa and Labetalol was the most common multidrug antihypertensive regimen used in $7.79 \%$ cases. Average number of antihypertensives per prescription was found to be 1.22 . A total of $83.76 \%$ prescriptions were found in generic name.

A study conducted on 60 pregnant women with blood pressure of $140 / 90 \mathrm{~mm} \mathrm{Hg}$ or more with $\geq 1+$ proteinuria between 20 and 38 weeks of gestation were randomly allocated to receive nifedipine $(n=20)$, methyldopa $(n=20)$ or labetalol $(n=20)$. Blood pressure was measured at $0,6,24,48$ and $72 \mathrm{~h}$ of initiation of antihypertensive drugs. Patients were also followed up for development of adverse drug effects during this period. Antihypertensive treatment with methyldopa was associated with reduction in systolic blood pressure (SBP) by $50 \mathrm{mmHg}$ and diastolic blood pressure (DBP) by $30 \mathrm{mmHg}$ at $72 \mathrm{~h}$. For the same period treatment with nifedipine was associated with reduction in SBP by $54 \mathrm{mmHg}$ and DBP by 30 $\mathrm{mmHg}$. Treatment with labetalol was associated with reduction in SBP by $70 \mathrm{mmHg}$ and DBP by $36 \mathrm{mmHg}$ at 72 h.Labetalol was more effective than methyldopa and nifedipine in controlling blood pressure in patients with pregnancy-induced hypertension while methyldopa and nifedipine are equally effective in controlling blood pressure.

A number of drugs in various combinations are generally used for effective long -term management of hypertension in pregnancy. There is need to survey the pattern of usage of anti hypertensives drugs, to see if the current usage is rational, effective and tolerated and in concordance with current guidelines for treatment of hypertension. The greatest challenge in treating hypertension in pregnancy is to reduce the blood pressure to assure the safety of mother and at same time not to compromise utero placental perfusion or cause harmful effects on the foetus. The ideal therapy of hypertension in pregnancy should be potent, rapidly acting and without any adverse maternal or foetal effect. The present study aims to investigate the drug utilization pattern of antihypertensive drugs in pregnancy induced hypertension.

Statement of the Problem: "Treatment of Pregnancy Induced Hypertension and Its Effect on Oxidative Stress"

\section{Objectives of the Study}

1. To assess the level of physiological parameters in blood of antenatal mothers with Pregnancy induced hypertension.

2. To monitor the treatment of antenatal mothers of Pregnancy induced hypertension.

3. To correlate the treatment of Pregnancy induced hypertension and its effects on oxidative stress with MDA and SOD estimation in antenatal mothers.

\section{Operational Definitions}

Oxidative Stress: - is essentially an imbalance between the production of free radicals and the ability of the body to counteract or detoxify their harmful effects through 
neutralization by antioxidants.

4. Pregnancy induced hypertension:-in this study, the antennal mothers, whose blood pressure is increased above 140/90, mmHg due to pregnancy.

5. Treatment of pregnancy induced hypertension:- in this study, the drugs given to antenatal mothers to reduce pregnancy induced hypertension.

Hypothesis: The study is based on the hypothesis: H1:-There is strong correlation between the treatments of pregnancy induced hypertension and oxidative stress.

Variables

Dependent- Oxidative stress in pregnancy induced hypertension.

Independent---Treatment of pregnancy induced hypertension.

\section{MATERIAL AND METHODS}

In this study Evaluative research approach was used. The research design was true experimental. The study will be conducted in AVBR hospital Sawangi (M), Wardha. Non probability convenient sampling technique will be used for this study.50 antenatal mothers upto 8 month, pregnant mothers in AVBR hospital, Sawangi (M), Wardha were the sample.

\section{Inclusion Criteria For Sampling}

1. Antenatal mothers with pregnancy induced hypertension.

2. Antenatal mothers admitted first time for pregnancy induced hypertension.

3. Antenatal mother's upto 8 months with pregnancy induced hypertension.

4. Willing to participate in the study.

5. Available at the time of study

\section{Exclusion Criteria}

1. Illness like anemia, diabetes mellitus, essential hypertension, renal insufficiency, cardiovascular disease which by themselves are known to alter free radical status.

Delimitation: The study is limited to the antenatal mothers after upto 8 months gestation with hypertension admitted in hospital.

\section{Discription of Tools}

Section A: Physiological Parameters in Blood of Antenatal Mothers.

Section B: Investigation Data (MDA and SOD estimation in antenatal mothers

\section{Procedure}

\section{At the time of admission-}

- Information about antenatal mother's name, age, obstratic history will be noted.

- Blood pressure and blood sample will be taken for MAD , and SOD estimation.
- $\quad$ Treatment for pregnancy induced hypertension will be recorded.

- $\quad$ Recording of data will be done.

2. At the time of discharge again the above procedure will be repeated.

- Blood Sample Collection

- $2 \mathrm{ml}$ blood will be collected in plain tubes for MDA estimation.

- $\quad 2 \mathrm{ml}$ blood will be collected in EDTA tubes for SOD estimation.

- Immediately the blood sample will be sent to the central research lab.

\section{Instrument}

1. Spectrophotometer

2. Weighing Balance

3. Centrifuge

4. Vortexer

5. Micropipette

\section{Procedure for Mda Estimation:}

1. Serum should be fresh and analyzed within 6 hours of separation.

2. Take three clean test tubes namely blank, standard and test.

3. To each test tube add 2 ul of Trichloroacetic Acid (TCA) solution.

4. To them add 800 ul of Thiobarbituric Acid (TBA) solution.

5. To the test tube blank add 200 ul distilled water.

6. To the test tube standard add 200 ul working MDA standard.

7. To the test tube add 200ul serum.

8. Final volume of solution will be $3 \mathrm{ml}$ in all the test tubes.

9. Then keep all the tubes in a boiling water bath for 20 minutes.

10. Then switch off the water bath and keep the tubes in water bath for further $10 \mathrm{~min}$.

11. After that cooling the tubes under tap water and centrifuge them at 300 RPM for $1 \mathrm{~min}$.

12. After that immediately take reading on uv- vis spectrophotometry at $531 \mathrm{~nm}$ and note the readings.

\section{Procedure for Sod Estimation:}

1. Take two clean tubes namely blank and test.

2. Take $2.7 \mathrm{ml}$ of tris buffer in a tube blank.

3. Take $2.699 \mathrm{ml}$ of tris buffer in a tube namely test and to it add 1 ul of hemolysate.

4. At the time of reading add 300ul pyrogallol to the test tube blank and start the stop watch simultaneously.

5. Final volume of solution will be $3 \mathrm{ml}$ in all the test tubes.

6. We have to take reading within $1 \mathrm{~min}$. vortex the mixture for $20 \mathrm{sec}$.

7. Put the solution in acuvette. Keep the cuvette in a spectrophotometer in a manner such that opaque side facing yourself. Do not touch the transparent side. 
8. After 60 sec. press enter, and again on 90 sec. press enter.

9. 10 readings come one by one which are shown on display screen. Note down the readings.
10. Take the readings for test in a same manner at 420nm.

\section{RESULTS}

\begin{tabular}{|c|c|c|c|}
\hline Parameters & $\begin{array}{l}\text { Pregnancy Induced } \\
\text { Hypertension }\end{array}$ & Preeclampsia & Eclampsia \\
\hline Age & 24.55 & 25.98 & 28.58 \\
\hline Mean gestational age in weeks & 36.8 & 32.65 & 30.16 \\
\hline $\begin{array}{l}\text { Mean systolic blood } \\
\text { pressure in } \mathrm{mm} \mathrm{Hg}\end{array}$ & 119.4 & 146.31 & 168.33 \\
\hline $\begin{array}{l}\text { Mean diastolic blood } \\
\text { pressure in } \mathrm{mm} \mathrm{Hg}\end{array}$ & 79.5 & 97.63 & 113.0 \\
\hline Proteinurea (mean) in gm/ day & Nil & 0.95 & 3.6 \\
\hline Oedema & Nil & Nil to + in all cases & ++ in all cases \\
\hline
\end{tabular}

Table 2. Comparison of MDA and SOD in pregnancy Induced Hypertension, Preeclampsia and Eclampsia

\begin{tabular}{|l|c|c|c|}
\hline & $\begin{array}{c}\text { Pregnancy } \\
\text { Induced } \\
\text { Hypertension }\end{array}$ & Preeclampsia & Eclampsia \\
\hline MDA (nmol/dl) & $109.09 \pm 24$ & $130.65 \pm 43.02$ & $141.04 \pm 50.95$ \\
\hline P-Value & $<0.001$ & $<0.001$ & $<0.001$ \\
\hline
\end{tabular}

Table 3

\begin{tabular}{|l|c|c|c|}
\hline & $\begin{array}{c}\text { Pregnancy } \\
\text { Induced } \\
\text { Hypertension }\end{array}$ & Preeclampsia & Eclampsia \\
\hline SOD & $3.24 \pm 1$. & $2.69 \pm 1.34$ & $2.59 \pm 1.33$ \\
\hline P-Value & $<0.001$ & $<0.001$ & $<0.001$ \\
\hline
\end{tabular}

MDA in pregnancy induced hypertension, preeclampsia and eclampsia was $109.09 \pm 24,130.65 \pm 43.02$ and $141.04 \pm 50.95$ respectively. . SOD in pregnancy induced hypertension, preeclampsia and eclampsia was $3.24 \pm 1,2.69 \pm 1.34$ and $2.59 \pm 1.33$ respectively.

\section{DISCUSSION}

The present study was planned to detect lipid peroxidation products i.e. MDA and SOD in PIH, pre eclampsia and eclampsia. The lipid peroxidation product like malondialdehyde (MDA) levels has been measured in plasma of hypertensive pregnant women. It was found that higher 02 free radical production, evidenced by increase levels of MDA in hypertensive pregnant women. The present study shows that, there is significant differencebetween $\mathrm{PIH}$, pre eclampsia and eclampsia regarding serum MDA.Rise in MDA could be due to increased generation ofROS due to the excessiveoxidative damage generated in the hypertensive patients. These 02 species in turn can oxidize many other importantbiomolecules including membrane lipids. The lipidperoxides \& free radicals may be important in pathogenesisof PIH. In similar previous study was done on pregnant women with pregnancy induced hypertension, it was found at there was a significant increase in erythrocytes MDA levels, activates of SOD, at GP level. 
A study conducted in Kerala, India evaluates the role of oxidative stress in PIH preeclampsia and eclampsia. This is done by measuring the levels of Malondialdehyde and superoxide dismutase in serum and catalase activity in erythrocytes. Age, gestational age, systolic and diastolic blood pressures and protein creatinine ratio in urine has also been included as baseline parameters. The results are tabulated in Tables 1-6. The mean value of MDA was found to be significantly increased in $(\mathrm{P}<0.001)$ in PIH group. Mean SOD and Catalase in PIH group was found to be significantly less $(\mathrm{P}<0.01)$ than that of the normal group. The mean values of MDA and Catalase in preeclampsia was found to be significantly increased $(\mathrm{P}<0.001)$ as compared to normal pregnant.

Whereas, the activity of SOD was decreased significantly $(\mathrm{P}<0.001)$ in patients suffering from preeclampsia. The result of MDA, SOD and Catalase was similar to preeclampsia in Eclampsia. When the serum MDA and catalase activity in erythrocytes in PIH, Preeclampsia and Eclampsia were compared with normal group has shown a significant increase $(\mathrm{P}<0.001)$ respectively in all the groups. Whereas, the activity of SOD in PIH, Preeclampsia and Eclampsia when compared with normal group has shown a significant decline $(\mathrm{P}<0.001)$ respectively in all the groups.

\section{CONCLUSION}

The present study has shown a significant increase in serum MDA levels, the indicator of lipid peroxidation, in the 3 groups suggest that lipid peroxidation plays a role in the pathogenesis of hypertensive disorders of pregnancy. Increased lipid peroxidation causes increased consumption of antioxidant free radical scavenging system. In PIH, preeclampsia and eclampsia there is an imbalance between lipid peroxides and the antioxidant system.

\section{REFERENCES}

Burton, G.J. and Jauniaux, E., 2004. Placental oxidative stress: from miscarriage to preeclampsia. Journal of the Society for Gynecologic Investigation, 11(6), pp.342352.

Jauniaux, E., Watson, A.L., Hempstock, J., Bao, Y.P., Skepper, J.N. and Burton, G.J., 2000. Onset of maternal arterial blood flow and placental oxidative stress: a possible factor in human early pregnancy failure. The American journal of pathology, 157(6), pp.2111-2122. Moffatt, 0., Drury, S., Tomlinson, M., Afnan, M. and Sakkas, D., 2002. The apoptotic profile of human cumulus cells changes with patient age and after exposure to sperm but not in relation to oocyte maturity. Fertility and sterility, 77(5), pp.1006-1011.

Poston, L., Chappell, L., Seed, P. and Shennan, A., 2011. Biomarkers of oxidative stress in pre-eclampsia. Pregnancy Hypertension: An International Journal of Women's Cardiovascular Health, 1(1), pp.22-27.

Sadraie, S.H., Saito, H., Kaneko, T., Saito, T. and Hiroi, M., 2000. Effects of aging on ovarian fecundity in terms of the incidence of apoptotic granulosa cells. Journal of assisted reproduction and genetics, 17(3), pp.168-173. Takahashi, T., Takahashi, E., Igarashi, H., Tezuka, N. and Kurachi, H., 2003. Impact of oxidative stress in aged mouse oocytes on calcium oscillations at fertilization. Molecular Reproduction and Development: Incorporating Gamete Research, 66(2), pp.143-152.

Wiener-Megnazi, Z., Vardi, L., Lissak, A., Shnizer, S., Reznick, A.Z., Ishai, D., Lahav-Baratz, S., Shiloh, H., Koifman, M. and Dirnfeld, M., 2004. Oxidative stress indices in follicular fluid as measured by the thermochemiluminescence assay correlate with outcome parameters in in vitro fertilization. Fertility and Sterility, 82, pp.1171-1176. 